\title{
Pancreatic Enzyme
}

National Cancer Institute

\section{Source}

National Cancer Institute. Pancreatic Enzyme. NCI Thesaurus. Code C18296.

Pancreatic enzymes are comprised primarily of proteases, lipase and amylase that catalyze the breakdown of fats, carbohydrates and proteins during the digestion of food.

However, the pancreas also produces many other digestive enzymes such as ribonuclease, deoxyribonuclease, gelatinase and elastase. 